\title{
Assessment of Image Quality for Optimal MRI Diagnostic Device Applied to Parameter Changes with 3D FRE at 1.5 T and 3.0 T
}

\author{
Eun-Hoe Goo \\ Department of Radiological Science, Cheongju University, Cheongju, Chungbuk,360- \\ 764, Republic of Korea \\ eunhoegoo@gmail.com
}

\begin{abstract}
The data analysis in this study was conducted to compare the advantages and disadvantages of the 1.5 T 3D TOF HSR method and the 3.0 T 3D TOF SR method, in order to determine whether $1.5 \mathrm{~T}$ can complement the image quality of intracranial vessels For SNRs and CNRs, significant results were obtained owing to the high scores of $3.0 \mathrm{~T}(\mathrm{p}<0.05)$. In the qualitative analysis, significant results were obtained for the A3, M3-M4, and P3-P4 segments owing to the high scores of $1.5 \mathrm{~T}$ ( $p<0.05)$. However, both $1.5 \mathrm{~T}$ and $3.0 \mathrm{~T} 3 \mathrm{D}$ FFE TOF methods provided images that allowed qualitative assessment. The findings of this study confirmed that 1.5 T 3D HRS MRI can complement 3.0 T 3D SR MRI.
\end{abstract}

Keywords: High spatial resolution, Standard resolution

\section{Introduction}

Diseases of the intracranial vessels include occlusion of the intravascular lumen and ruptures of blood vessels due to embolism or thrombosis. According to Statistics Korea, about 3,000 people died per 100,000 of those aged 65 or more, and $11.6 \%$ of the deaths were caused by stroke in 2011. Although this is a lower percentage than the death rate from cancer, stroke ranked as the first cause of death for single organ diseases [1]. MRI methods include noninvasive MRA methods such as 2D TOF (2D time of flight), 3D TOF, 2D PC (2D phase contrast), and 3D PC(Eq. 1-4),

$$
\begin{gathered}
\emptyset=\gamma \int x(t) g(t) d t=\gamma \int\left(x_{0}+v t\right) g(t) d t=\varphi_{s}+\varphi_{m}, \\
\varphi_{m}=\gamma \int v G t d t \\
S_{1=} S_{s}+S_{m}, S_{2}=S_{s}+S_{m} e^{j k m} \\
\varphi_{m}=\arg \left(S_{2}\right)-\arg \left(\frac{S_{2}}{S_{1}}\right)=\arg \left(\frac{S_{2}}{S_{1}^{*}}\right)=\gamma \int v G t d t .
\end{gathered}
$$

$\emptyset:$ phase shift, $\varphi_{s}:$ static spin, $\varphi_{m}:$ moving spin, $S$ : singal intensity, $v$ : velocity,

\footnotetext{
${ }^{1}$ Article history:

Received (May 15, 2019), Review Result (June 11, 2019), Accepted (August 6, 2019) 
G: gradient strenth, t: time.

As well as the invasive CE MRA (contrast enhanced magnetic resonance angiography) method, which can depict blood vessels using a contrast medium [2][3]. High-resolution 3.0 T MRI is often being conducted in and outside the country as magnetic resonance intracranial vessel angiography for intracranial vessel diseases, thanks to its high signal strength and microvessel detection rate [4]. However, the technique cannot be applied to all patients because the number of installed machines is small. Therefore, 1.5 T HSR (high spatial resolution) and 3.0 T SR (standard resolution) methods, which have been tested clinically for data analysis, were here compared in order to determine whether 1.5 T MRI can complement 3.0 T MRI in terms of image quality in the 3D TOF test of intracranial vessels.

\section{Materials and Methods}

\subsection{Subjects}

Among the patients who visited our hospital between October 2012 and January 2015 and received the TOF MRA test using $1.5 \mathrm{~T}$ or $3.0 \mathrm{~T}, 300$ patients were randomly selected $(1.5 \mathrm{~T}$ : 150 subjects, $3.0 \mathrm{~T}: 150$ subjects), and their data were analyzed through the PACS network. The subjects consisted of 156 men and 144 women aged between 30 and 80, with a mean age of $61.26 \pm 3$.

\subsection{Testing Method}

No contrast media were used for the analyzed data. The test machines were $1.5 \mathrm{~T}$ and $3.0 \mathrm{~T}$ MRIs (Philips, Medical System, Achieva, The Netherlands). For the received coil, a 16 channel head coil was used. For pulse sequence employed in the analysis, 3D FFE was used for gradient magnetic field echo, which depicts blood vessels well. The acquired images were reconstructed as MIP (maximum intensity projection) before being sent to the PACS network.

\subsection{Analysis method}

Then quantitative analysis of SNR (signal to noise ratio, Eq. 10) and CNR (contrast to noise ratio) was conducted for the internal carotid artery (ICA), middle cerebral artery (MCA, M1), anterior cerebral artery (ACA, A1), anterior cerebral artery (ACA, A2), and vertebral artery (VA). For qualitative analysis, the degrees of depiction for middle cerebral arteries (M1, M2, M3, M4), posterior cerebral arteries (P1, P2, P3, P4), and anterior cerebral arteries (A1, A2, A3) were classified into three levels. These levels were described as: no vessel segments are shown (poor, 1), vessel segments are vaguely shown (good, 2), and vessel segments are clearly shown (excellent, 3 ).

\subsection{Statistics Analysis}

For the quantitative analysis of the two machines, the statistical significance for SNR and CNR was calculated by independent sample t-test. Furthermore, Mann-Whitney U test was applied to the general blood vessel depiction, including the circle of wills.

\section{Results}

For the average SNRs for ICA, M1, A1, A2, and VA in the two machines, the values of 3.0T were higher (1.5T: $4654.4 \pm 226.3$, 3.0T: $6444.2 \pm 237.6)$. Furthermore, $3.0 \mathrm{~T}$ also obtained higher values for the average CNRs of vessels and surrounding tissues (1.5T: $3201.2 \pm 61.6$, 
3.0T: $4488.4 \pm 38.9$ ). For the depiction of segments for A1, A2, and A3, in the view of the observer, the 3.0 T SR method obtained higher scores (1.5 T: $2.70 \pm 0.08,3.0 \mathrm{~T}: 2.74 \pm 0.06)$, but the differences were insignificant ( $p>0.05)$. For the depictions of M1 (2.66 \pm 0.47 vs. 2.86 $\pm 0.35)$ and $\mathrm{M} 2(2.56 \pm 0.50$ vs. $2.86 \pm 0.35)$, the $3.0 \mathrm{~T} \mathrm{SR}$ method also obtained slightly higher scores, but the differences were insignificant ( $p>0.05)$. For the depictions of M3 ( $2.87 \pm 0.35$ vs. $2.83 \pm 0.37)$ and M4 (2.86 \pm 0.35 vs. $2.63 \pm 0.49)$, the 1.5 T HSR method obtained slightly higher scores, and the differences were significant $(\mathrm{p}<0.05)$. Figure 1 shows visualizations of the 1.5 T HSR and 3.0 T SR methods. The 1.5 T HSR method obtained significantly superior values when compared to the 3.0 T SR method for small vessels such as C (M3, M4) and E (P3, $\mathrm{P} 4)$. When visually assessed by observer, the signal intensities of $\mathrm{B}$ images, including the VA vessel of F, were higher than those of the A images for ICA, M1, A1, and A2 vesse.
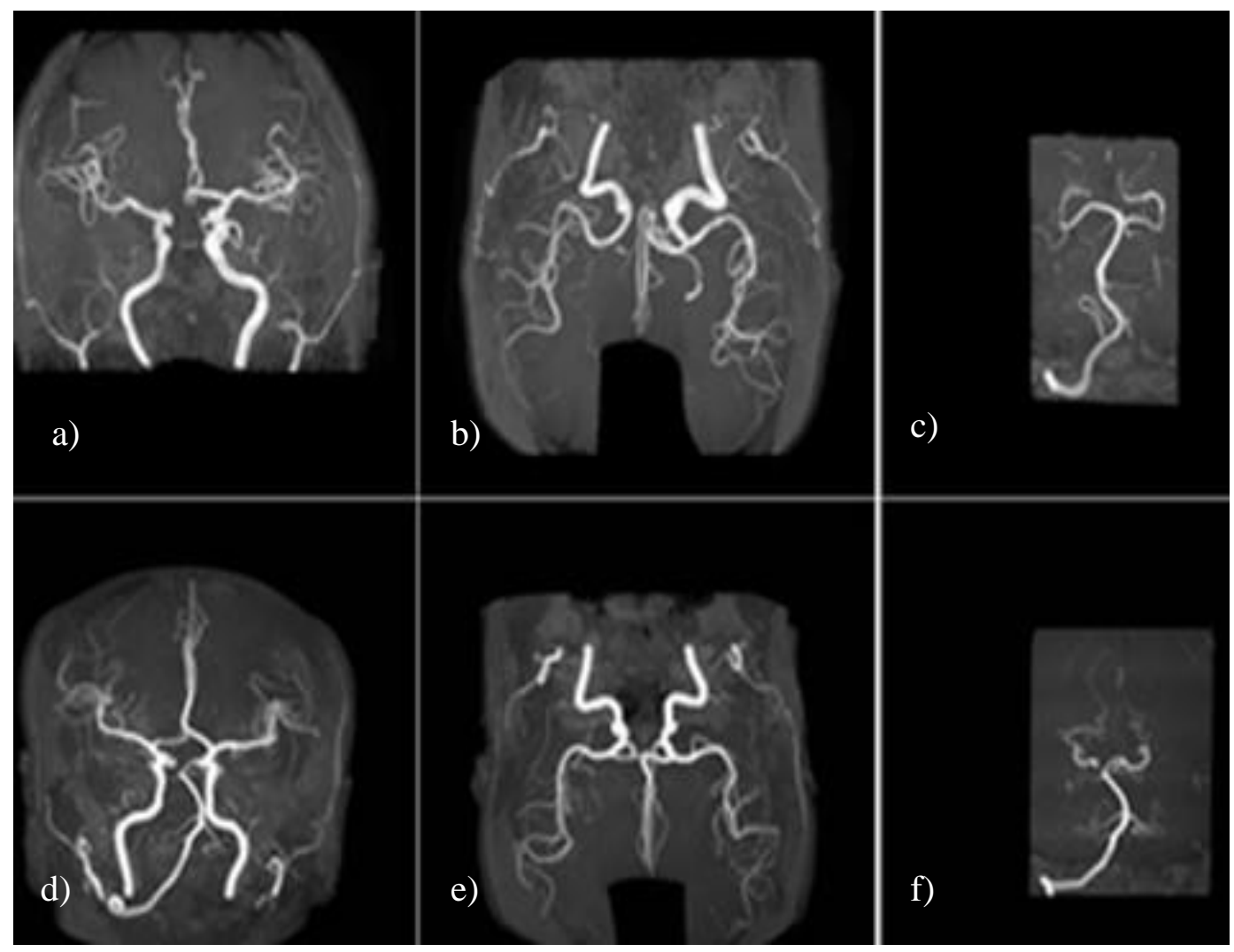

Figure 1. 3D TOF maximum intensity projection(MIP) images of $1.5 \mathrm{~T}(\mathrm{a}, \mathrm{b}, \mathrm{c})$ and 3.0 T(d,e,f). Note that vessel segments such as M3, M4, P3, P4 are better visualized at $1.5 \mathrm{~T}$ HSR imaging than at $3.0 \mathrm{~T}$ SR imaging.

\section{Discussion}

The 3D FFE TOF pulse sequence is generally tested for intracranial vessels in tertiary medical centers, including general hospitals. Recently, many 3.0 T MRI machines have been installed and provide much clinical information, but they have the disadvantage that artifacts are generated owing to the effects of high magnetic field strength [5][6][7]. Among the artifacts, susceptibility artifact is proportional to the main magnetic field (B0) and TE (echo to time), but it is inversely proportional to bandwidth. In the case of chemical shift, artifacts, fat, and water 
repeated in-out of phase over a constant TE time. In general, there is a chemical shift of 3.5 ppm between these two tissues, and, owing to the difference in frequency between $220 \mathrm{~Hz}$ of $1.5 \mathrm{~T} \mathrm{MRI}$ and $440 \mathrm{~Hz}$ of $3.0 \mathrm{~T}$ MRI, the resonance of fat occurred at frequencies lower by $220 \mathrm{~Hz}$ and $440 \mathrm{~Hz}$ than that of water. When applying 3D FFE TOF pulse sequence in $1.5 \mathrm{~T}$ or 3.0 T MRI, the opposed phase TE value, fat suppression, and MT methods were analyzed for optimum depiction of blood vessels. Dielectric resonance artifacts[8] also clearly appeared in this data analysis. Figure 2 compares the volume data and MIP image for the dielectric resonance artifact in $1.5 \mathrm{~T}$ and $3.0 \mathrm{~T}$ units.

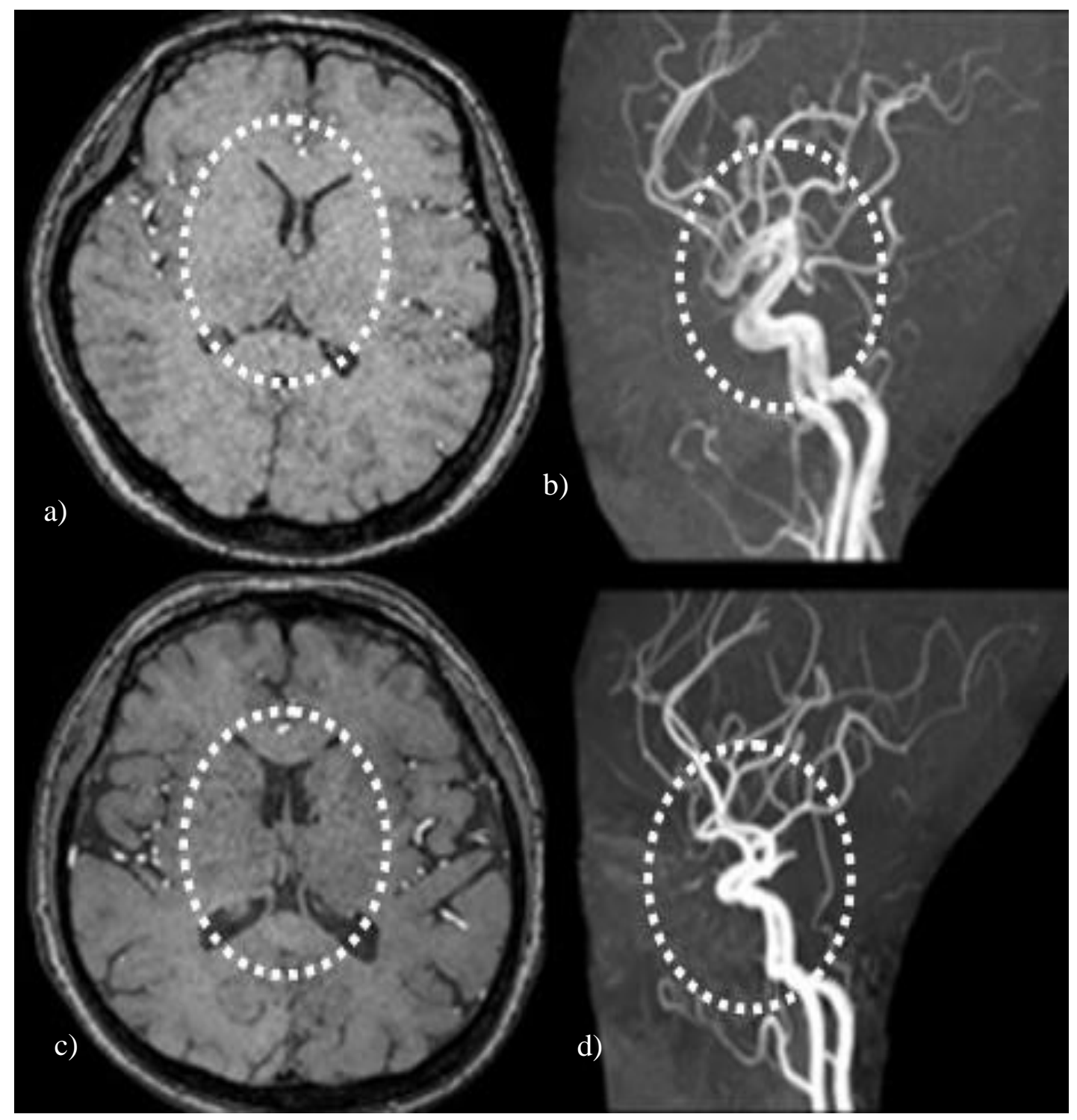

Figure 2. Axial 3D FFE TOF volume(a, c) and $\operatorname{MIP}(b, d)$ images at $1.5 \mathrm{~T}(\mathrm{a}, \mathrm{b})$ and 3.0 T(c, d, bright areas ) units.

The 3.0 T MRI test using FFE pulse sequence improved the image quality when large vessels, hemorrhage, and cartilage were tested, but it was limited in the depiction of small vessels owing 
to susceptibility artifacts. In the data analysis of this study, too, because of susceptibility artifacts, the 1.5 T HSR method obtained better results than the 3.0 T SR method for the M3M4 and P3-P4 segments Figure 3.

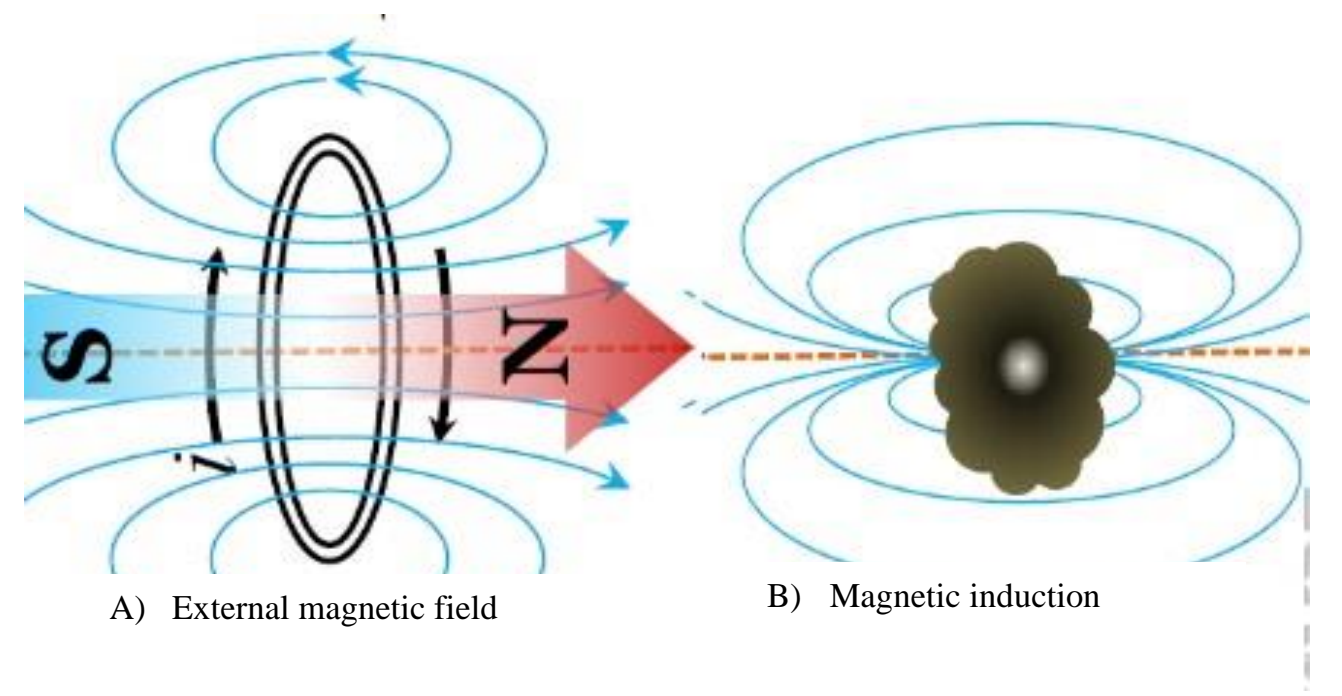

Figure 3. Magnitude of magnetic field strength.

$\mathrm{H}=$ external magnetic field(A); A magnetic field, either induced or permanent, generates a magnetic force, also called magnetic field strength. B: magnetic induction(B); Magnitude of internal magnetic field strength within a material exposed to an $\mathrm{H}$ field, also called magnetic flux density.

\section{Conclusion}

In conclusion, the application of methods to the two machines for this data analysis generated a clear difference in image information. In the quantitative analysis, the 3.0 T MRI showed high SNR and CNR values. However, in the qualitative analysis, the $1.5 \mathrm{~T}$ MRI acquired high scores in A3, M3, M4, P3, and P4, and generally depicted well the small vessels in the outer side of the brain. The 1.5 T MRI machine had the disadvantage of long test time, but it obtained images with uniformity in signal intensity. The 3.0 T MRI machine had the disadvantage of short test time, but it obtained images with non-uniform signal intensities. Therefore, this data analysis showed that, when performing a TOF 3D test of intracranial vessels, the $1.5 \mathrm{~T}$ MRI machine can be used in place of the 3.0 T MRI machine in settings that do not have it.

\section{References}

[1] D. Mozaffarian, E. J. Benjamin, A. S. Go, D. K. Arnett, M. J. Blaha, M. Cushman, S. R. Das, S. de Ferranti, J. P. Després, H. J. Fullerton, V. J. Howard, M. D. Huffman, C. R. Isasi, M. C. Jiménez, S. E. Judd, B. M. Kissela, J. H. Lichtman, L. D. Lisabeth, S. Liu, R. H. Mackey, D. J. Magid, D. K. McGuire, C. S. Moy, P. Muntner, M. E. Mussolino, K. Nasir, R. W. Neumar, G. Nichol, L. Palaniappan, D. K. Pandey, M. J. Reeves, C. J. Rodriguez, W. Rosamond, P. D. Sorlie, "Executive Summary: Heart Disease and Stroke Statistics-2016 Update", Circulation. Vol.133, No.4, pp.447-454 (2016). DOI: 10.1161/CIR.0000000000000366 
[2] J. Attali, A. Benaissa, S. Soize, K. Kadziolka, C. Portefaix, L. Pierot, "Follow-up of intracranial aneurysms treated by flow diverter: comparison of three-dimensional time-of-flight MR angiography (3D-TOF-MRA) and contrast-enhanced MR angiography (CE-MRA) sequences with digital subtraction angiography as the gold standard", Journal of NeuroInterventional Surgery. Vol.8, No.1, (2014). DOI: 10.1136/neurintsurg-2014011449

[3] P. Volonghi, D. Tresoldi, M. Cadioli, A. M. Usuelli, R. Ponzini, U. Morbiducci, A. Esposito, G. Rizzo, "Automatic extraction of three - dimensional thoracic aorta geometric model from phase contrast MRI for morphometric and hemodynamic characterization", Magnetic Resonance in Medicine, Vol.75, No.2, pp.873882. (2016). DOI: $10.1002 / \mathrm{mrm} .25630$

[4] Z. J. Jia, R. Zhao, Z. G. Yang, Q. H. Huang, X. Q. Deng, B. Hong, J. M. Liu, "Intracranial atherosclerotic middle cerebral arterial stenosis research based on 3.0 Tesla highresolution magnetic resonance imaging: recent progress”, J South Med Univ, Vol.35, No.1, pp.154-158, (2015). DOI: 10.3969/j.issn.1673-4254.2015.01.32

[5] M. A. Bernstein, "Field Strength Dependence in MRI : Advantages and Artifacts at 3 T", ISMRM, (2006), pp. $1-8$.

[6] B. L. Schmitz, A. J. Aschoff, M. H. Hoffmann, G. Grön, "Advantages and pitfalls in 3T MR brain imaging: a pictorial review", American Journal of Neuroradiology. Vol.26, No.9, pp.2229-2237 (2005).

[7] J. N. Oshinski, J. G. Delfino, P. Sharma, A. M. Gharib, R. I. Pettigrew, "Cardiovascular magnetic resonance at 3.0T: Current state of the art”, Journal of Cardiovascular Magnetic Resonance, (2010). DIO: 10.1186/1532429X-12-55

[8] C. M. Collins, W. Liu, W. Schreiber, Q. X. Yang, M. B. Smith, "Central brightening due to constructive interference with, without, and despite dielectric resonance”, Journal of Magnetic Resonance Imaging. Vol.21, No.2, pp.192-196 (2005). DOI: 10.1002/jmri.20245

\section{Authors}

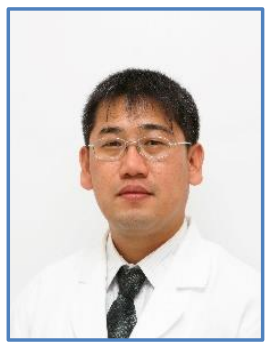

Eun-Hoe Goo

2012. 02:

Department of Electronic Physics(Doctor of science), Soonchunhyang University, Graduate School.

2015. 04: Currently:

Department of Radiological Science, Cheongju

University, Assistant Professor. 